\title{
Isomerization and fluorescence depolarization of merocyanine 540 in polyacrylic acid. Effect of $p H$
}

\author{
DIPANKAR SUKUL, SOBHAN SEN, PARTHA DUTTA and \\ KANKAN BHATTACHARYYA* \\ Physical Chemistry Department, Indian Association for the Cultivation of \\ Science, Jadavpur, Kolkata 700 032, India \\ e-mail: pckb@mahendra.iacs.res.in
}

MS received 26 April 2002; revised 8 July 2002

\begin{abstract}
Dynamics of isomerization and fluorescence depolarization of merocyanine 540 (MC540) in an aqueous solution of polyacrylic acid (PAA) have been studied using picosecond time resolved fluorescence spectroscopy. It is observed that the dynamics of isomerization and depolarization are sensitive enough to monitor the uncoiling of PAA at high $p \mathrm{H}(>6)$. At low $p \mathrm{H}(<3)$, when the polymer remains in a hypercoiled form, polymer bound MC540 experiences very high microscopic friction and, hence, the isomerization and depolarization processes are very slow. At high $p \mathrm{H}(>6)$ a polyanion is formed and the polymer assumes an extended configuration due to electrostatic repulsion. At high $p \mathrm{H}(>6)$, the anionic probe MC540 is expelled from the polyanion to bulk water and the dynamics of isomerization and fluorescence depolarization become faster by 12 and 5 times respectively, compared to those at low $p \mathrm{H}$.
\end{abstract}

Keywords. Photoisomerization; fluorescence depolarization; uncoiling.

\section{Introduction}

Many chemical processes involve motion of a portion of the molecule against another (e.g. in isomerization) or the molecule as a whole (e.g. in reorientation). Such processes are affected by the friction of the medium ${ }^{1}$. While effect of friction in bulk liquid is known, there is much less information on friction in organized environments. The microscopic friction of organized assemblies towards photoisomerization and reorientational dynamics has been the subject of several recent studies. Photoisomerization i.e. cis-trans isomerization in excited electronic state, has been studied in a ${\text { cyclodextrin } \text { cavity }^{2} \text {, at the water surface }}^{3,4}$, in the water pool of microemulsion ${ }^{5}$, at micellar interface ${ }^{6}$, lipids ${ }^{7}$, sol-gel glass ${ }^{8}$ and polymer environments ${ }^{9}$. Reorientational dynamics has been studied using fluorescence anisotropy decay in DNA ${ }^{10}$, micelles ${ }^{11-13}$, polymer-surfactant aggregates ${ }^{14}$. In the case of reorientational dynamics, the motion of the macromolecules becomes superimposed on the bound fluorescence probes. Several authors have discussed in detail how one can delineate the motion of macromolecules or surfactant assemblies and the fluorescent probe ${ }^{10-14}$.

\footnotetext{
*For correspondence
} 
In the present work, we focus on the effect of $p \mathrm{H}$-induced structural change of polyacrylic acid on isomerization and reorientation. The major aim is to demonstrate that the dynamics of isomerization and reorientation is a sensitive probe to monitor this structural change. For acidic polyelectrolytes such as polyacrylic acid (PAA), polymethacrylic acid (PMAA), DNA or poly-glutamic acid, at low $\mathrm{pH}$ the acidic (-COOH) groups do not dissociate. At low $p \mathrm{H}$, the polyacids remain in very compact conformations with hydrophobic microenvironments. In such conformations, these polymers resemble globular proteins and can encapsulate hydrophobic solutes. At high $p \mathrm{H}$, the $-\mathrm{COOH}$ group becomes ionized. Because of the repulsion between the anionic carboxylate side chains, the polymer assumes an extended rod-like shape. At low $p \mathrm{H}$, a fluorescent probe experiences an extremely hydrophobic environment with very large friction inside the hypercoiled PAA polymer. However, at high $p \mathrm{H}$, when the polyanion PAA opens up due to electrostatic repulsion, the fluorescent probe becomes exposed to water and experiences bulk water-like environment with low friction. The $p \mathrm{H}$-dependent conformational transition of such polymers have been studied by fluorescence ${ }^{9,15-19}$, atomic force microscopy ${ }^{19}$, and has been theoretically analysed using the Ising model ${ }^{17}$. Previous fluorescence studies of this interesting phenomenon relied on the steady state intensities of the vibronic bands of pyrene ${ }^{17,18}$, electron quenching of aromatic singlets ${ }^{15 \mathrm{~b}}$ and the efficiency of energy transfer ${ }^{\mathrm{g}, 20}$.

The $p \mathrm{H}$-dependent uncoiling of the polymers is likely to affect the dynamics of photophysical processes, e.g. cis-trans isomerization and fluorescence depolarization due to friction. Jones and Oh studied photoisomerization of a cyanine dye, pseudo-isocyanine iodide in PMMA using the phase modulation technique ${ }^{9 a}$. The main nonradiative pathway of cyanine dyes is photoisomerization about a central double bond ${ }^{1,21-24}$. Jones and $\mathrm{Oh}$ reported that at low $p \mathrm{H}$ when the microscopic friction is high, the photoisomerization is suppressed ${ }^{9 a}$. This is manifested in the increase in the emission intensity and lifetime at low $p \mathrm{H}^{\text {9a }}$. However, they did not provide the rate constants of isomerization of the dye bound to PMMA at different $p \mathrm{H}$. The rate of fluorescence anisotropy decay also depends on microscopic friction. The uncoiling of PAA with increase in $p \mathrm{H}$ should reduce microscopic friction towards reorientational motion. Jones and Oh did not study the orientational dynamics of PMMA bound dye.

In the present work, we have chosen another cyanine dye, merocyanine 540 (MC540, chart 1) as a probe ${ }^{25-29}$. The main non-radiative pathway for MC540 is photoisomerization, as the other common non-radiative pathways, e.g. intersystem crossing to the triplet manifold and the internal conversion, are extremely inefficient ${ }^{26 a, b}$. The ground state conformation of MC540 is exclusively all-trans ${ }^{26 \mathrm{~b}}$. Following excitation to the first singlet state, the system moves freely along the torsional coordinate as there is practically no barrier for cis-trans isomerization in the excited state. The potential surface of this system is such that the perpendicular geometry in the $\pi \pi^{*}$ excited state is almost isoenergetic with the ground state ${ }^{\text {la }}$. Thus at the perpendicular geometry, it undergoes rapid transition from the excited electronic state to the nearly isoenergetic ground state. In the ground state, the perpendicular geometry corresponds to the peak of the barrier between the cis and the trans isomer in the ground state. Once the system reaches the peak it can undergo transition to either of them in the ground state with equal probability. The dynamics of isomerization and fluorescence depolarization of MC540 has earlier been used to probe many organized media such as micelles, reverse micelles and proteins ${ }^{11,27-30}$. 


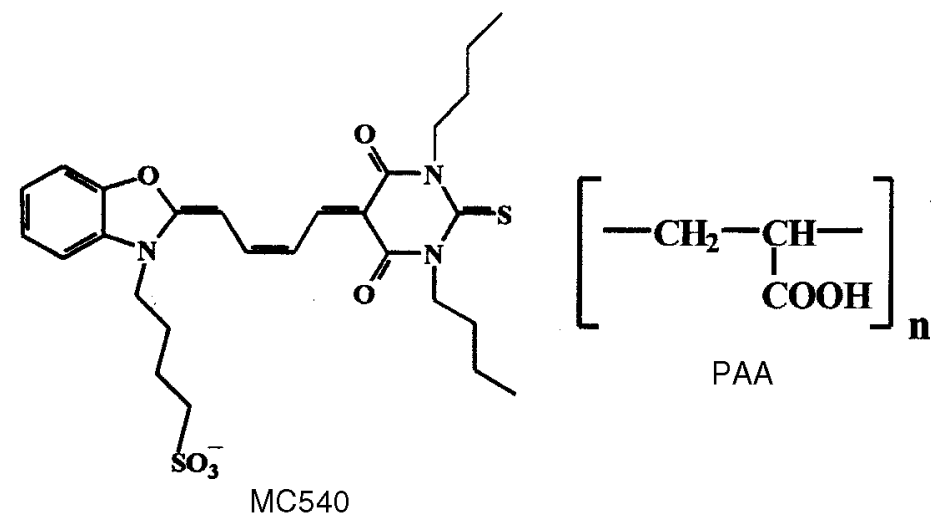

Chart 1. Structures of MC540 and PAA (acid form).

In this work we plan to investigate how the variation of friction in PAA with $p \mathrm{H}$ affects the dynamics of isomerization and fluorescence depolarization of MC540.

\section{Experimental}

The dye, merocyanine 540 (Sigma) and the polymer, PAA, sodium salt (Aldrich, $\left.M_{w}=5100\right)$ were used as received. The sample was excited at $570 \mathrm{~nm}$ using a synchronously pumped rhodamine 6G dye laser (Coherent 702-1) pumped by a CW mode locked Nd:YAG laser (Antares 76S). The exciting laser light is cut off by a Corning filter (2-60). The fluorescence at $630 \mathrm{~nm}$ was detected at magic angle polarization, using a MCP photomultiplier (Hamamatsu 2809U). For rotational relaxation studies, the polarization of the exciting light was rotated by $90^{\circ}$ at regular intervals using a half-wave plate to get the perpendicular $\left(I_{\perp}\right)$ and parallel $\left(I_{\|}\right)$components. Then the anisotropy function, $r(t)$ was calculated using the formula,

$$
r(t)=\frac{I_{\|}(t)-G I_{\perp}(t)}{I_{\|}(t)+2 G I_{\perp}(t)} .
$$

The $G$ value of the setup was determined using a probe with very fast rotational relaxation time, e.g. nile red in methanol.

\section{Results}

\subsection{Steady state results}

In aqueous solution, MC540 exhibits two broad absorption peaks at $500 \mathrm{~nm}$ and $535 \mathrm{~nm}^{28,29}$. The broad absorption peak at $500 \mathrm{~nm}$ has been assigned to the nonfluorescent dimer of MC540 and the peak at $535 \mathrm{~nm}$ to the monomer ${ }^{28,29}$. The absorption spectra of an aqueous solution containing $4 \times 10^{-6} \mathrm{M}$ MC540 and $10 \mathrm{mM}$ PAA at different $p \mathrm{H}$ values are shown in figure 1a. The individual contributions of the dimer and the monomer to the total absorption spectrum are obtained by decomposing the absorption spectrum. By decomposing the absorption spectrum of MC540 in $10 \mathrm{mM}$ 
(a)

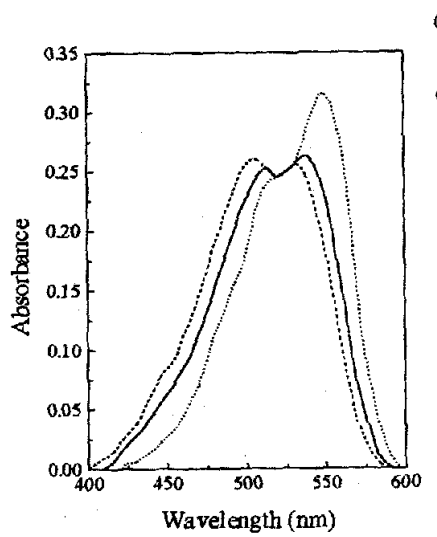

(b)

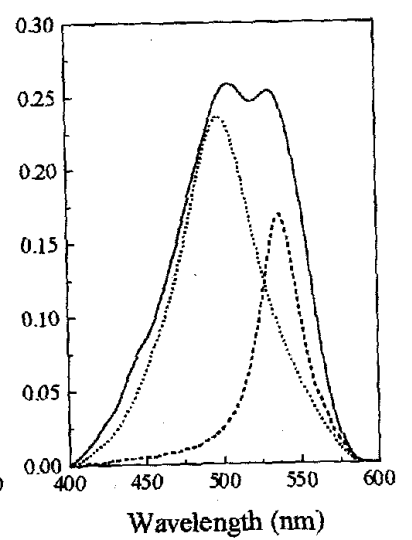

(c)

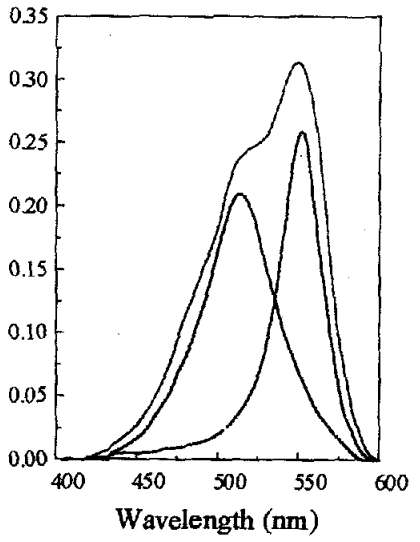

Figure 1. (a) Absorption spectra of an aqueous solution containing $4 \times 10^{-6} \mathrm{M}$ MC540 and $10 \mathrm{mM}$ PAA at (i) $p \mathrm{H}=6(---)$, (ii) $p \mathrm{H}=4(-)$, (iii) $p \mathrm{H}=2(\cdots \cdots)$. (b) Decomposition of the absorption spectra of merocyanine 540 in $10 \mathrm{mM}$ PAA at $p \mathrm{H}=6$, dimer $(\cdots \cdots)$, monomer (----), total $(-)$. (c) Decomposition of the absorption spectra of merocyanine 540 in $10 \mathrm{mM}$ PAA at $p \mathrm{H}=2$, dimer $(\cdots \cdots)$, monomer $(----)$, total $(-)$.

PAA at $p \mathrm{H}=6$, two bands are located at $500 \mathrm{~nm}$ and $535 \mathrm{~nm}$ (figure $1 \mathrm{~b}$ ). Evidently the $500 \mathrm{~nm}$ absorption band may be attributed to the dimer of MC540 and the $535 \mathrm{~nm}$ band to the monomer. At $p \mathrm{H}=2$, the decomposed absorption spectrum of MC540 in $10 \mathrm{mM}$ PAA exhibits two absorption bands at $510 \mathrm{~nm}$ and $550 \mathrm{~nm}$ (figure 1c). We have earlier shown that the monomer absorption band exhibits a red shift with decrease in solvent polarity ${ }^{28,29}$. While absorption maximum of the monomer is at $535 \mathrm{~nm}$ in water, it shifts to $565 \mathrm{~nm}$ in dioxane ${ }^{29}$. Thus the $550 \mathrm{~nm}$ peak at $p \mathrm{H}=2$ is assigned to MC540 monomer bound to relatively less polar and hydrophobic environment of neutral PAA.

It is readily seen that at $p \mathrm{H}=6$, the height of dimer absorption peak of MC540 is nearly 1.4 times that of the monomer peak (figure $1 \mathrm{~b}$ ). However, at $p \mathrm{H}=2$, the monomer peak becomes 1.25 times that of the dimer peak (figure 1c). This shows that with decrease in $p \mathrm{H}$, inside the PAA the dimers of MC540 breakup and polymer-bound MC540 exists predominantly as a monomer. In summary, absorption spectra suggest that at low $p \mathrm{H}$, when MC540 molecules are entrapped inside the hypercoiled conformation of PAA, the dye molecules remain largely as monomers.

With decrease in solvent polarity, MC540 exhibits a red shift in emission maximum and a marked increase in emission quantum yield $\left(\phi_{f}\right)$ and lifetime ${ }^{29}$. Emission maximum of MC540 shifts from $575 \mathrm{~nm}$ in water to $590 \mathrm{~nm}$ in dioxane. $\phi_{f}$ of MC540 increases from 0.04 in water to 0.52 in dioxane. Figure 2 shows the variation in the emission spectra of MC540 with $p \mathrm{H}$ in $10 \mathrm{mM}$ PAA. It is readily seen, with increase in $p \mathrm{H}$, that the emission maximum exhibits a blue shift from $585 \mathrm{~nm}$ at $p \mathrm{H}=3$ to $575 \mathrm{~nm}$ at $p \mathrm{H}=6$. It may be noted that in aqueous solution, the emission spectrum of $4 \times 10^{-6}$ M MC540 does not change with the variation in $p \mathrm{H}$.

With increase in $p \mathrm{H}$, the emission quantum yield $\left(\phi_{f}\right)$ of MC540 in $10 \mathrm{mM}$ PAA decreases nearly 7 times from 0.28 at $p \mathrm{H}=2$ to 0.04 at $p \mathrm{H} 7$ (figure $2 \mathrm{a}$ and table 1). The 
(a)

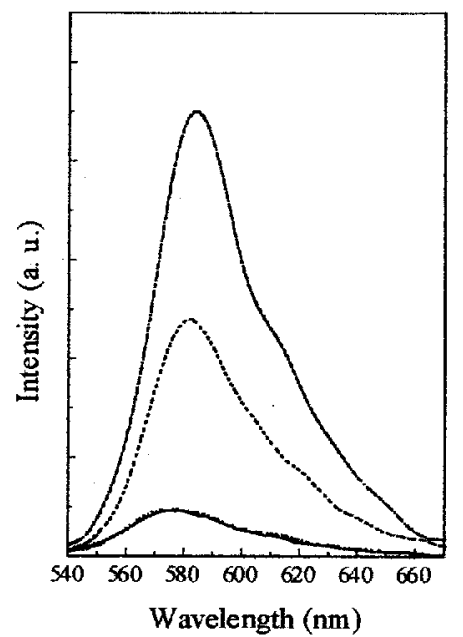

(b)

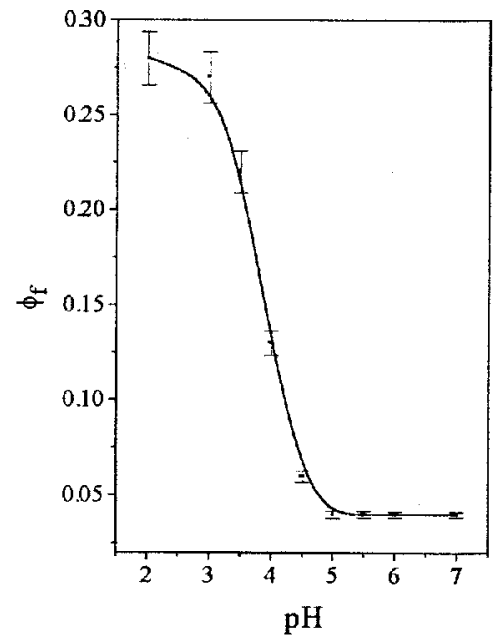

Figure 2. (a) Emission spectra of an aqueous solution containing $4 \times 10^{-6} \mathrm{M}$ MC540 and $10 \mathrm{mM}$ PAA at (i) $p \mathrm{H}=6$ (一), (ii) $p \mathrm{H}=5$ (…), (iii) $p \mathrm{H}=4$ (----) and (iv) $p \mathrm{H}=3(-\cdot-\cdot)$. (b) $p \mathrm{H}$ dependence quantum yield $\left(\phi_{f}\right)$ of MC540 in an aqueous solution containing $4 \times 10^{-6} \mathrm{M}$ MC540 and $10 \mathrm{mM}$ PAA.

Table 1. Variation of quantum yield $\left(\phi_{f}\right)$, average life time $\left(\tau_{f}\right)$ and isomerization rate constant $\left(k_{\text {iso }}\right)$ of merocyanine 540 (MC 540) with change in $p \mathrm{H}$ in an aqueous solution of $10 \mathrm{mM}$ polyacrylic acid (PAA).

\begin{tabular}{lccc}
\hline$p \mathrm{H}$ & $\phi_{f}^{*}$ & $\left\langle\tau_{f}\right\rangle^{*}(\mathrm{ps})$ & $k_{\text {iso }}\left(\times 10^{-9}\right)\left(\mathrm{s}^{-1}\right)$ \\
\hline $2 \cdot 0$ & $0 \cdot 28$ & 1000 & $0 \cdot 72$ \\
$3 \cdot 0$ & $0 \cdot 27$ & 975 & $0 \cdot 75$ \\
$3 \cdot 5$ & $0 \cdot 22$ & 900 & $0 \cdot 87$ \\
$4 \cdot 0$ & $0 \cdot 13$ & 700 & $1 \cdot 25$ \\
$4 \cdot 5$ & $0 \cdot 06$ & 350 & $2 \cdot 70$ \\
$5 \cdot 0$ & $0 \cdot 04$ & 170 & $5 \cdot 65$ \\
$5 \cdot 5$ & $0 \cdot 04$ & 130 & $7 \cdot 40$ \\
$6 \cdot 0$ & $0 \cdot 04$ & 120 & $8 \cdot 00$ \\
$7 \cdot 0$ & $0 \cdot 04$ & 110 & $8 \cdot 70$ \\
\hline
\end{tabular}

$* \pm 5 \%$

variation of $\phi_{f}$ of MC540 with $p \mathrm{H}$ in $10 \mathrm{mM}$ PAA is summarized in table 1 and displayed in figure $2 \mathrm{~b}$. From figure $2 \mathrm{~b}$ it is apparent that the emission quantum yield of MC540 displays a sigmoidal dependence on $p \mathrm{H}$, shows sharp decrease at $p \mathrm{H}>4$ and levels off at $p \mathrm{H} \approx 5$. The marked increase in $\phi_{f}$ of MC540 in $10 \mathrm{mM} \mathrm{PAA}$ at low $p \mathrm{H}$ suggests significant retardation of the non-radiative pathways of MC540. As noted earlier, the main nonradiative pathway in the excited state of MC540 is isomerization. The increase in $\phi_{f}$ at low $p \mathrm{H}$ indicates that the isomerization process of MC540 is markedly suppressed when it is trapped inside the 'hypercoiled' undissociated acid, PAA. 


\subsection{Time resolved fluorescence}

In aqueous solution, fluorescence decay of MC540 is a single exponential with a lifetime $\left(\tau_{f}\right)$ of $110 \mathrm{ps}$ and independent of $p \mathrm{H}$ of the solution ${ }^{29}$. The fluorescence decay of MC540 in $10 \mathrm{mM}$ PAA differs markedly from that in pure water. We fitted the decays to a biexponential, e.g. $a_{1} \exp \left(-t / \tau_{1}\right)+a_{2} \exp \left(-t / \tau_{2}\right)$ and used the average fluorescence lifetime, $\left\langle\tau_{f}\right\rangle=a_{1} \tau_{1}+a_{2} \tau_{2}$.

Fluorescence decays of MC540 in $10 \mathrm{mM}$ PAA at different $p \mathrm{H}$ values are shown in figure $3 \mathrm{a}$ and the average fluorescence lifetimes $\left(\left\langle\tau_{f}\right\rangle\right)$ are listed in table 1. Figure $3 \mathrm{~b}$ shows variation of $\left\langle\tau_{f}\right\rangle$ with $p \mathrm{H}$. It is readily seen that with increase in $p \mathrm{H}, \tau_{f}$ of MC540 decreases about 9 times from $1000 \mathrm{ps}$ at $p \mathrm{H}=2$ to a bulk water like value of $110 \mathrm{ps}$ at $p \mathrm{H}>6$. The increase in $\left\langle\tau_{f}\right\rangle$ at low $p \mathrm{H}$ provides additional support to the contention that the isomerization process of MC540 is inhibited inside the hypercoiled PAA. With increase in $p \mathrm{H}$, as the polymer uncoils and becomes negatively charged, it repels the anionic probe MC540 to bulk water. As a result, the dynamics of isomerization becomes faster and becomes very similar to that in bulk water.

From the observed values of $\phi_{f}$ and $\left\langle\tau_{f}\right\rangle$, the rate constant $\left(k_{\text {iso }}\right)$ of the nonradiative isomerization process of MC540 has been determined using the relation,

$$
k_{\text {iso }}=\left(1-\phi_{f}\right) /\left\langle\tau_{f}\right\rangle .
$$

The values of $k_{\text {iso }}$ of MC540 in a $10 \mathrm{mM}$ aqueous solution of PAA at various $p \mathrm{H}$ levels are given in table 1. Variation of $k_{\text {iso }}$ of MC540 as a function of $p \mathrm{H}$ is shown in figure 3c. It is obvious that the rate constant of the isomerization process increases about 12 times as $p \mathrm{H}$ is increased from 2 to 7 . The plot of $k_{\text {iso }}$ against $p \mathrm{H}$ exhibits a sharp transition at a $p \mathrm{H} \approx 4 \cdot 5$. The remarkably small magnitude of $k_{\text {iso }}$ of MC540 in PAA at low $p \mathrm{H}$, may be attributed to the restrictions imposed on the isomerization process of MC540 by the polymer chains in the hypercoiled form.

\subsection{Fluorescence depolarization}

The rotational or reorientational motion of a fluorescent probe gives rise to fluorescence depolarization. As a result, decay of the fluorescence anisotropy function, $r(t)$ is a measure of the rotational mobility of the probe. In water, the decay of fluorescence anisotropy of MC540 is found to be a single exponential with the time constant $600 \pm 30 \mathrm{ps}$. This is similar to the reported anisotropy decay of MC540 in ethanol ${ }^{11}$ and dioxane ${ }^{30}$. The anisotropy decay of MC540 in water was found to be independent of $p \mathrm{H}$.

In the presence of $10 \mathrm{mM}$ PAA, fluorescence anisotropy decay of MC540 is found to be very different from that in pure water. At low $p \mathrm{H}$, fluorescence anisotropy decay is found to be biexponential with much longer time constants (figure 4 and table 2). For instance at $p \mathrm{H}=2$, anisotropy decay of MC540 exhibits two components of $\tau_{1 R}=1500 \mathrm{ps}$ $\left(a_{1 R}=0.33\right.$, i.e. $\left.33 \%\right)$ and $\tau_{2 R}=4000 \mathrm{ps}\left(a_{2 R}=0.67\right.$, i.e. $\left.67 \%\right)$. Thus in $10 \mathrm{mM}$ PAA at $p \mathrm{H}=2$, the average rotational relaxation time of MC540, $\left\langle\tau_{\text {rot }}\right\rangle=a_{1 R} \tau_{1 R}+a_{2 R} \tau_{2 R}$, is $3200 \pm 150$ ps. This is about 5 times slower than the rotational relaxation time of MC540 in water (600 ps). At $p \mathrm{H} \geq 6$, the anisotropy decay of MC540 in $10 \mathrm{mM}$ PAA is found to be identical to that in pure water. The plot of $\left\langle\tau_{\text {rot }}\right\rangle$ of MC540 in $10 \mathrm{mM}$ PAA against $p \mathrm{H}$ exhibits a sigmoidal dependence with sharp transition at $p \mathrm{H} \approx 4$ (figure 5). The very long rotational relaxation time of MC540 in PAA at low $p \mathrm{H}$, indicates that the rotational mobility of the probe decreases markedly when the polymer wraps around the probe. 
(a)

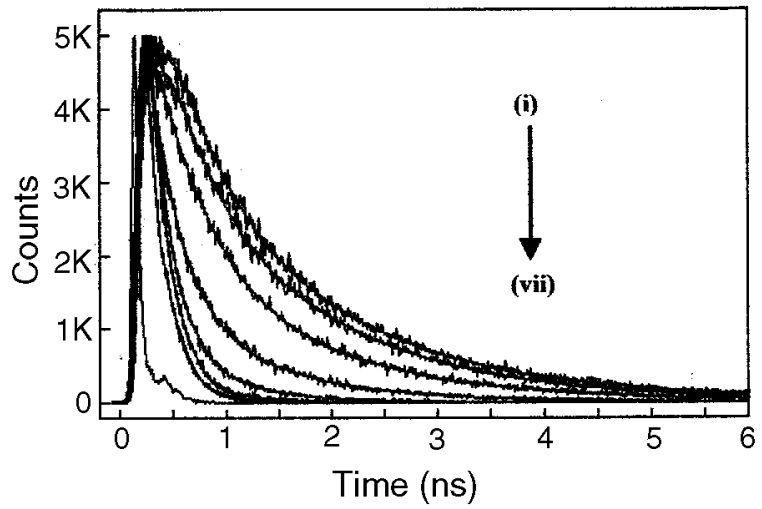

(b)

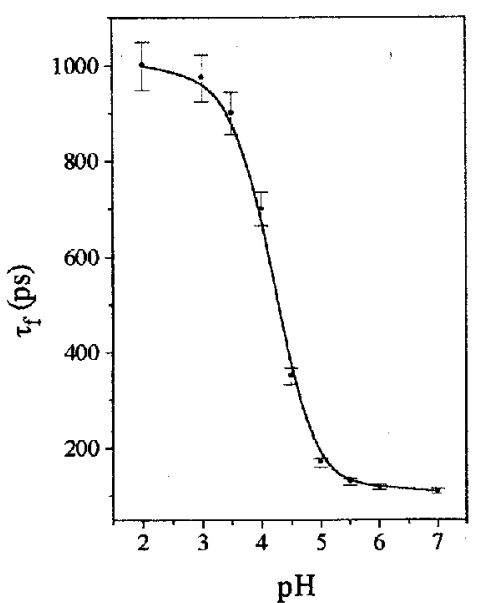

(c)

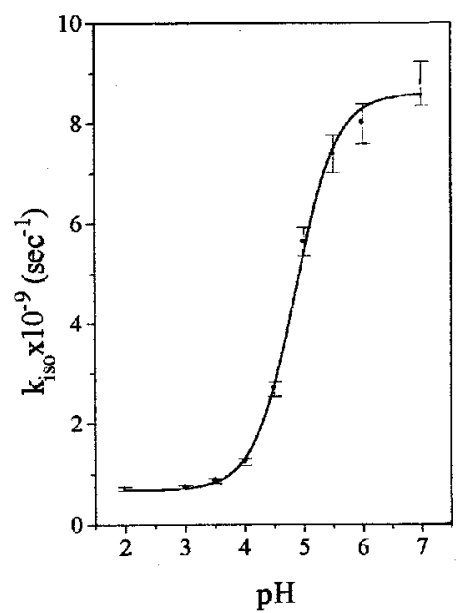

Figure 3. (a) Fluorescence decays of MC540 in an aqueous solution containing $4 \times 10^{-6} \mathrm{M}$ MC540 and $10 \mathrm{mM}$ PAA at (i-vii) $p \mathrm{H}=2 \cdot 0,3 \cdot 0,3 \cdot 5,4 \cdot 0,4 \cdot 5,5 \cdot 5$ and $7 \cdot 0$. (b) Variation of average fluorescence lifetime $\left(\left\langle\tau_{f}\right\rangle\right)$ of MC540 with $p \mathrm{H}$ in $10 \mathrm{mM}$ PAA. (c) $p \mathrm{H}$ dependence $k_{\text {iso }}$ of MC540 in $10 \mathrm{mM}$ PAA.

Table 2. Variation of fluorescence anisotropy decay time $\left(\tau_{\text {rot }}\right)$ of merocyanine 540 (MC540) with change of $p \mathrm{H}$ in a $10 \mathrm{mM}$ polyacrylic acid solution.

\begin{tabular}{llrlcc}
\hline$p \mathrm{H}$ & $a_{1 R}$ & $\tau_{1 R}(\mathrm{ps})$ & $a_{2 R}$ & $\tau_{2 R}(\mathrm{ps})$ & $\left\langle\tau_{\mathrm{rot}}{ }^{*}(\mathrm{ps})\right.$ \\
\hline $2 \cdot 0$ & $0 \cdot 33$ & 1500 & $0 \cdot 67$ & 4000 & 3200 \\
$3 \cdot 0$ & $0 \cdot 15$ & 500 & $0 \cdot 85$ & 3600 & 3100 \\
$3 \cdot 5$ & $0 \cdot 12$ & 450 & $0 \cdot 88$ & 3100 & 2800 \\
$4 \cdot 0$ & $0 \cdot 13$ & 140 & $0 \cdot 87$ & 2400 & 2100 \\
$4 \cdot 5$ & $0 \cdot 18$ & 170 & $0 \cdot 82$ & 1800 & 1500 \\
$5 \cdot 0$ & $0 \cdot 30$ & 250 & $0 \cdot 70$ & 1500 & 1100 \\
$5 \cdot 5$ & $0 \cdot 50$ & 150 & $0 \cdot 50$ & 1400 & 780 \\
$6 \cdot 0$ & $1 \cdot 0$ & 600 & & & 600 \\
$7 \cdot 0$ & $1 \cdot 0$ & 600 & & & 600 \\
\hline
\end{tabular}

$*\left\langle\tau_{\text {rot }}\right\rangle=a_{1 R} \tau_{1 R}+a_{2 R} \tau_{2 R} ; \pm 5 \%$ 
(a)

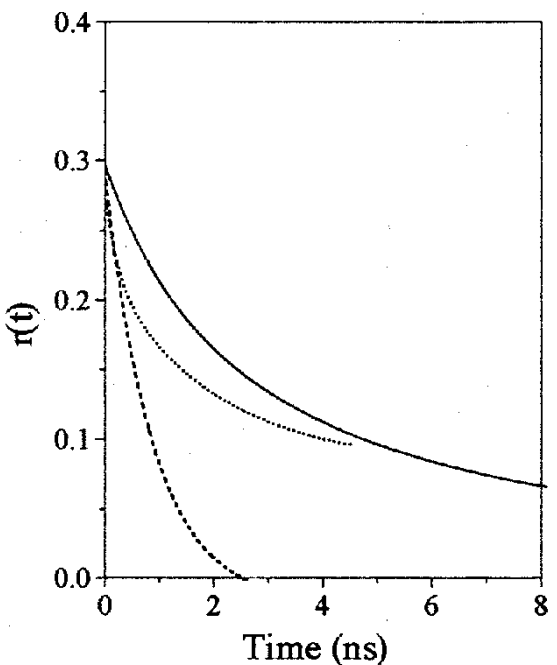

(b)

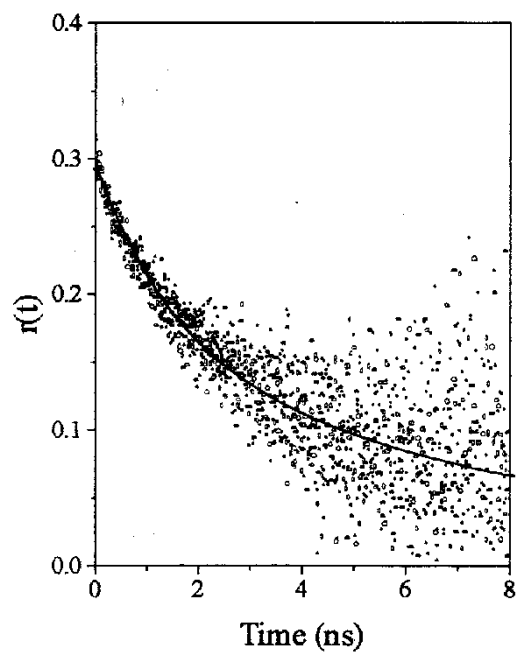

Figure 4. (a) Decays of fluorescence anisotropy of MC540 in an aqueous solution containing $4 \times 10^{-6} \mathrm{M}$ MC540 and $10 \mathrm{mM}$ PAA at (i) $p \mathrm{H}=7$ (---), (ii) $p \mathrm{H}=5(\cdots \cdots)$ and (iii) $p \mathrm{H}=2(-)$. (b) Fitted curve along with raw data of fluorescence anisotropy decay of MC540 in $10 \mathrm{mM}$ PAA at a $p \mathrm{H}=2$.

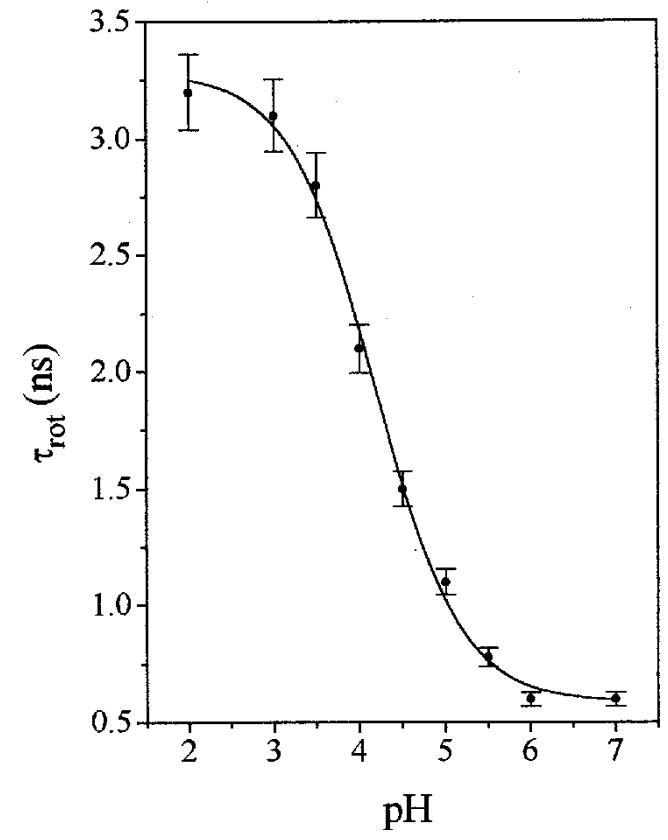

Figure 5. $\quad p \mathrm{H}$ dependence of average rotational relaxation time $\left(\left\langle\tau_{\text {rot }}\right\rangle\right)$ of MC540 in an aqueous solution containing $4 \times 10^{-6} \mathrm{M}$ MC540 and $10 \mathrm{mM}$ PAA. 


\section{Discussions}

The most important finding of the present work is that the $p \mathrm{H}$-dependent structural change (uncoiling) of the polymer PAA, significantly influences the dynamics of isomerization and reorientation of MC540. At low $p \mathrm{H}$, when the polymer wraps around the probe MC540 molecule in a compact hypercoiled form, the dynamics of the two processes are markedly slowed down compared to bulk water. However, at high $p \mathrm{H}$ the polyanion expands and because of its electrostatic repulsion the anionic probe, MC540, is expelled from the hydrophobic pocket of the polymer to bulk water. As a result, at high $p \mathrm{H}$ the probe $\mathrm{MC540,}$, exhibits bulk water-like fast isomerization and reorientational dynamics. For both the processes, the $p \mathrm{H}$ dependence of all the dynamic parameters displays a sigmoidal curve with a sharp transition at $p \mathrm{H} \approx 4$. It may be recalled that similar sharp transition at $p \mathrm{H} \approx 4$ was also reported in other studies on $\mathrm{PAA}^{9,15-20}$, and has been attributed to ionization of PAA and consequent uncoiling. Thus, isomerization and reorientational dynamics are sensitive probes to monitor the $p \mathrm{H}$ dependent uncoiling of the polymer.

In $10 \mathrm{mM}$ PAA, the dynamics of isomerization of MC540 at low $p \mathrm{H}$ is found to be 12 times slower than that at high $p \mathrm{H}(>6)$ or that in water. It has been demonstrated earlier that for MC540 the photoisomerization dynamics depends on polarity as well as friction $^{29}$. For PAA, however, it has been shown that even at low $p \mathrm{H}$ the polarity of the microenvironment remains quite high ${ }^{17}$. The observed emission maximum $(585 \mathrm{~nm})$ of MC540 in $10 \mathrm{mM}$ PAA corresponds to a water-dioxane mixture containing $60 \%$ water $^{29}$. For this mixture, the average lifetime $\left(\left\langle\tau_{f}\right\rangle\right)$ and quantum yield $\phi_{f}$ of MC540 are $350 \mathrm{ps}$ and 0.2 respectively ${ }^{29}$. However, in the present work for MC540 in $10 \mathrm{mM} \mathrm{PAA}$ at $p \mathrm{H}=2$ we observed a higher $\phi_{f}(\approx 0 \cdot 28)$ and longer lifetime $(\approx 1000 \mathrm{ps})$. This indicates that the observed retardation of the isomerization process of MC540 in PAA at low $p \mathrm{H}$ arises only partly due to reduced polarity and mainly from the high microscopic friction in the hypercoiled polymer.

The very high microscopic friction in PAA at low $p \mathrm{H}$ is also responsible for the slow rotational relaxation time of MC540 at low $p \mathrm{H}$. The observed multiexponential decay of $r(t)$ for MC540 in PAA at low $p \mathrm{H}$ may arise from various factors ${ }^{31}$. First, in such a micro-heterogeneous system, the probe MC540 might experience different magnitudes of friction in different regions. Second, the motion of the polymer chains may become superimposed on the rotational motion of the probe MC540 and as a result, the decay of $r(t)$ may be due to different kinds of independent motions ${ }^{10-14}$. In the case of spherical micelles, the multiexponential decay of $r(t)$ is analysed in terms of three independent motions - (i) wobbling of the probe in a cone; (ii) translational diffusion of the probe along the spherical micellar particles, and (iii) overall rotation of the micelle ${ }^{10-14}$. In the present case of the hypercoiled PAA at low $p \mathrm{H}$ shape, hydrodynamic radius, location of the probe and many other parameters are not known. Thus quantitative analysis of the decay of $r(t)$ is difficult at this stage. Nevertheless, it is evident that the average rotational relaxation time of MC540 in PAA, very clearly indicates the uncoiling of the polymer with increase in $p \mathrm{H}$. One should note that the measurement of microscopic friction (microviscosity) is not straightforward even in the case of simple liquids ${ }^{1,13,14}$. Even the dependence of translational diffusion coefficient on viscosity is found to be nonlinear in many cases ${ }^{32}$. Because of these complications, many authors have used reorientatioal relaxation time $\left(\tau_{\text {rot }}\right)$ as a measure of microscopic friction. One can also use isomerization 
rate as a measure of microviscosity. Thus both the plots of $\tau_{\text {rot }}$ vs $p \mathrm{H}$ or $k_{\text {iso }}$ vs $p \mathrm{H}$ should be considered as a plot of microscopic friction vs $p \mathrm{H}$.

\section{Conclusion}

Dynamics of isomerization and fluorescence depolarization (i.e. rotational motion) of MC540 are found to be sensitive indicators to monitor the change of microenvironment of polyacrylic acid as a function of $p \mathrm{H}$. With increase in $p \mathrm{H}$ as the polymer (PAA) undergoes a structural change from hypercoiled to extended rod-like configuration, the rate constant of photoisomerization of MC540 increases about 12 times as $p \mathrm{H}$ of the solution increases from 2 to 7 . This leads to decrease of emission intensity and lifetime of MC540 by similar magnitude. The decrease in friction with $p \mathrm{H}$ is also manifested in the nearly 5-fold decrease in the average rotational relaxation time of MC540 in PAA.

\section{Acknowledgements}

Thanks are due to the Council of Scientific and Industrial Research (CSIR), New Delhi and Department of Science and Technology, Government of India for generous research grants. DS, SS and PD thank the CSIR for fellowships.

\section{References}

1. (a) Waldeck D H 1991 Chem. Rev. 91 415; (b) Bagchi B and Oxtoby D W 1983 J. Chem. Phys. 78 2735; (c) Bagchi B 1989 Annu. Rev. Phys. Chem. 40115

2. Duveneck G L, Sitzman E V, Eisenthal K B and Turro N J 1989 J. Phys. Chem. 937166

3. Eisenthal K B 1996 Chem. Rev. 961343

4. Shi X, Borguet E, Tarnovsky A N and Eisenthal K B 1996 Chem. Phys. 205167

5. Datta A, Pal S K, Mandal D and Bhattacharyya K 1997 Chem. Phys. Lett. 27877

6. (a) Pal S K, Datta A, Mandal D and Bhattacharyya K 1998 Chem. Phys. Lett. 288 793; (b) Greiser F, Lay M and Thistlewaite P J 1985 J. Phys. Chem. 892065

7. (a) Holmes A S, Birch D J S, Sanderson A and Aloisi G C 1997 Chem. Phys. Lett. 266 309; (b) Bernik D, Tymczyszn E, Daraio M E and Negri R M 1999 Photochem. Photobiol. 7040

8. Casalboni M, Matteis F De, Ferone V, Prosposito P, Senesi R, Pizzoferrato R, Bianco A and De Mico A 1998 Chem. Phys. Lett. 291167

9. (a) Jones G II and Oh C 1994 J. Phys. Chem. 98 2367; (b) Jones G II and Rahman M A 1992 Chem. Phys. Lett. 200241

10. Miller D P, Robbins R J and Zewail A H 1982 J. Chem. Phys. 762080

11. Quitevis E L, Marcus A H and Fayer M D 1993 J. Phys. Chem. 975762

12. Wittouck N W, Negri R M and De Schryver F C 1994 J. Am. Chem. Soc. 11610601

13. Maiti N C, Krishna M M G, Britto P J and Periasamy N 1997 J Phys. Chem. B101 11051

14. Sen S, Sukul D, Dutta P and Bhattacharyya K 2001 J. Phys. Chem. A105 7495

15. (a) Webber S E 1998 J. Phys. Chem. B102 2618; (b) Stramel D, Webber S E and Rogers M A 1989 J. Phys. Chem. 93 1928; (c) Teng Y, Morrison M E, Munk P, Webber S E and Prochazka K 1998 Macromolecules 31 3578; (d) Eckert A R, Martin T J and Webber S E 1997 J. Phys. Chem. A101 1646

16. Chen G, Ito Y and Imanishi Y 1997 Macromolecules 307001

17. Philippova O E, Hourdet D, Audebert R and Khokhlov A R 1997 Macromolecules 308278

18. Olea A F and Thomas J K 1989 Macromolecules 221165

19. Kathman E E L, White L A and McCormik C L 1997 Macromolecules 305297

20. (a) Morawetz H 1996 Macromolecules 29 2689; (b) Horsky J and Morawetz H 1988 Makromol. Chem. 1892475

21. Velsko S R and Fleming G R 1982 Chem. Phys. 6559

22. Jaraudis J $1980 \mathrm{~J}$. Photochem. 1335 
23. Hicks J M, Vandersall M T, Sitzmann E V and Eisenthal K B 1987 Chem. Phys. Lett. 135413

24. Hicks J M, Vandersall M T, Babarogic Z and Eisenthal K B 1985 Chem. Phys. Lett. 11618

25. Vedamuthu M, Singh S, Onganer O, Bessire D R, Yin M, Quitevis E L and Robinson G W 1996 J. Phys. Chem. 10011907

26. (a) Onganer Y, Yin M, Bessire D R and Quitevis E L 1993 J. Phys. Chem. 97 2344; (b) Benniston A C, Gulliya K S and Harriman A 1994 J. Chem. Soc., Faraday Trans. 90953

27. Khazraji A C, Hotchandani S, Das S and Kamath P V 1999 J. Phys. Chem. B103 4693

28. (a) Verkman A S 1987 Biochemistry 26 4050; (b) Drassten P R and Webb W W 1978 Biochemistry 175228

29. Mandal D, Pal S K, Sukul D and Bhattacharyya K 1999 J. Phys. Chem. A103 8156

30. Flom S R and Fendler J H 1988 J. Phys. Chem. 925908

31. (a) Toptygin D, Svoda J, Konopasek I and Brand L 1992 J. Chem. Phys. 96 7919; (b) James D R and Ware W R 1985 Chem. Phys. Lett. 120 485; (c) Cho C H, Chung M, Lee J, Nguyen T, Singh S, Vedamuthu M, Yao S, Zhu S-B and Robinson G W 1995 J. Phys. Chem. 997806

32. (a) Bowmann R M and Eisenthal K B 1989 Chem. Phys. Lett. 155 99; (b) Zwanzig R and Harrison A K 1985 J. Chem. Phys. 835861 